$\mathrm{J}$ o u r n a l of

Mathematics

and Applications

JMA No 38, pp 85-104 (2015)

\title{
Approximate controllability of the impulsive semilinear heat equation ${ }^{1}$
}

\author{
Hugo Leiva and Nelson Merentes
}

Abstract: In this paper we apply Rothe's Fixed Point Theorem to prove the interior approximate controllability of the following semilinear impulsive Heat Equation

$$
\left\{\begin{array}{lr}
z_{t}=\Delta z+1_{\omega} u(t, x)+f(t, z, u(t, x)), & \text { in } \quad(0, \tau] \times \Omega, t \neq t_{k} \\
z=0, & \text { on } \quad(0, \tau) \times \partial \Omega, \\
z(0, x)=z_{0}(x), & x \in \Omega, \\
z\left(t_{k}^{+}, x\right)=z\left(t_{k}^{-}, x\right)+I_{k}\left(t_{k}, z\left(t_{k}, x\right), u\left(t_{k}, x\right)\right), & x \in \Omega
\end{array}\right.
$$

where $k=1,2, \ldots, p, \Omega$ is a bounded domain in $\mathbb{R}^{N}(N \geq 1), z_{0} \in L_{2}(\Omega), \omega$ is an open nonempty subset of $\Omega, 1_{\omega}$ denotes the characteristic function of the set $\omega$, the distributed control $u$ belongs to $C\left([0, \tau] ; L_{2}(\Omega)\right)$ and $f, I_{k} \in$ $C([0, \tau] \times \mathbb{R} \times \mathbb{R} ; \mathbb{R}), k=1,2,3, \ldots, p$, such that

$$
\begin{gathered}
|f(t, z, u)| \leq a_{0}|z|^{\alpha_{0}}+b_{0}|u|^{\beta_{0}}+c_{0}, \quad u \in \mathbb{R}, z \in \mathbb{R} . \\
\left|I_{k}(t, z, u)\right| \leq a_{k}|z|^{\alpha_{k}}+b_{k}|u|^{\beta_{k}}+c_{k}, \quad k=1,2,3, \ldots, p, \quad u \in \mathbb{R}, z \in \mathbb{R} .
\end{gathered}
$$

with $\frac{1}{2} \leq \alpha_{k}<1, \frac{1}{2} \leq \beta_{k}<1, \quad k=0,1,2,3, \ldots, p$. Under this condition we prove the following statement: For all open nonempty subsets $\omega$ of $\Omega$ the system is approximately controllable on $[0, \tau]$. Moreover, we could exhibit a sequence of controls steering the nonlinear system from an initial state $z_{0}$ to an $\epsilon$ neighborhood of the final state $z_{1}$ at time $\tau>0$.

AMS Subject Classification: primary: 93B05; secondary: $93 C 10$.

Keywords and Phrases: impulsive semilinear heat equation, approximate controllability, Rothe's fixed point Theorem.

${ }^{1}$ This work has been supported by CDCHT-ULA-C-1796-12-05-AA and BCV

COPYRIGHT (c) by Publishing Department Rzeszów University of Technology P.O. Box 85, 35-959 Rzeszów, Poland 


\section{Introduction}

There are many practical examples of impulsive control systems, a chemical reactor system with the quantities of different chemicals serve as the states, a financial system with two state variables of the amount of money in a market and the saving rates of a central bank and the growth of a population diffusing throughout its habitat is often modeled by reaction-diffusion equation, for which much has been done under the assumption that the system parameters related to the population environment, either are constant or change continuously.However, one may easily visualize situations in nature where abrupt changes such as harvesting, disasters and instantaneous stoking may occur. This observation motivates us to study the approximate controllability of the following Semilinear Impulsive Heat Equation

$$
\left\{\begin{array}{lr}
z_{t}=\Delta z+1_{\omega} u(t, x)+f(t, z, u(t, x)), & \text { in } \quad(0, \tau] \times \Omega, t \neq t_{k} \\
z=0, & \text { on } \quad(0, \tau) \times \partial \Omega \\
z(0, x)=z_{0}(x), & x \in \Omega \\
z\left(t_{k}^{+}, x\right)=z\left(t_{k}^{-}, x\right)+I_{k}\left(t_{k}, z\left(t_{k}, x\right), u\left(t_{k}, x\right)\right), & x \in \Omega
\end{array}\right.
$$

where $k=1,2, \ldots, p, \Omega$ is a bounded domain in $\mathbb{R}^{N}(N \geq 1), z_{0} \in L_{2}(\Omega), \omega$ is an open nonempty subset of $\Omega, 1_{\omega}$ denotes the characteristic function of the set $\omega$,the distributed control $u$ belongs to $C\left([0, \tau] ; L_{2}(\Omega)\right)$ and $f, I_{k} \in C([0, \tau] \times \mathbb{R} \times \mathbb{R} ; \mathbb{R})$, $k=1,2,3, \ldots, p$, such that

$$
\begin{gathered}
|f(t, z, u)| \leq a_{0}|z|^{\alpha_{0}}+b_{0}|u|^{\beta_{0}}+c_{0}, \quad u, z \in \mathbb{R} . \\
\left|I_{k}(t, z, u)\right| \leq a_{k}|z|^{\alpha_{k}}+b_{k}|u|^{\beta_{k}}+c_{k}, \quad k=1,2,3, \ldots, p, \quad u, z \in \mathbb{R} . \\
\frac{1}{2} \leq \alpha_{k}<1, \frac{1}{2} \leq \beta_{k}<1, \quad k=0,1,2,3, \ldots, p,
\end{gathered}
$$

and

$$
z\left(t_{k}, x\right)=z\left(t_{k}^{+}, x\right)=\lim _{t \rightarrow t_{k}^{+}} z(t, x), \quad z\left(t_{k}^{-}, x\right)=\lim _{t \rightarrow t_{k}^{-}} z(t, x) .
$$

In almost all reference on impulsive differential equations the natural space to work in is the Banach space

$$
\begin{aligned}
& P C([0, \tau] ; Z) \\
& =\left\{z: J=[0, \tau] \rightarrow Z: z \in C\left(J^{\prime} ; Z\right), \exists z\left(t_{k}^{+}, \cdot\right), z\left(t_{k}^{-}, \cdot\right) \quad \text { and } \quad z\left(t_{k}, \cdot\right)=z\left(t_{k}^{+}, \cdot\right)\right\},
\end{aligned}
$$

where $Z=L_{2}(\Omega)$ and $J^{\prime}=[0, \tau] \backslash\left\{t_{1}, t_{2}, \ldots, t_{p}\right\}$, endowed with the norm

$$
\|z\|=\sup _{t \in[0, \tau]}|z(t, \cdot)| z
$$

with

$$
\|z\|_{Z}=\sqrt{\int_{\Omega}\|z(x)\|^{2} d x}, \quad \forall z \in Z=L_{2}(\Omega) .
$$


Definition 1.1 (Approximate Controllability) The system (1.1) is said to be approximately controllable on $[0, \tau]$ if for every $z_{0}, z_{1} \in Z=U=L_{2}(\Omega), \varepsilon>0$ there exists $u \in C([0, \tau] ; U)$ such that the solution $z(t)$ of (1.1) corresponding to $u$ verifies:

$$
z(0)=z_{0} \quad \text { and } \quad\left\|z(\tau)-z_{1}\right\|_{Z}<\varepsilon, \quad(\text { Fig.2) }
$$

where

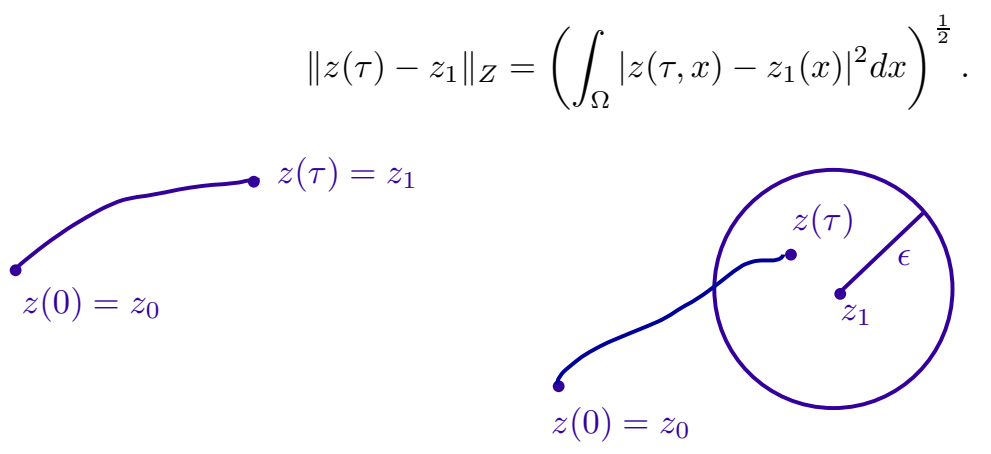

Fig.1

Fig. 2

Definition 1.2 (Controllability to Trajectories) The system (1.1) is said to be controllable to trajectories on $[0, \tau]$ if for every $z_{0}, \hat{z}_{0} \in Z=U=L_{2}(\Omega)$ and $\hat{u} \in$ $C([0, \tau] ; U)$ there exists $u \in C([0, \tau] ; U)$ such that the mild solution $z(t)$ of (1.1) corresponding to $u$ verifies:

$$
z\left(\tau, z_{0}, u\right)=z\left(\tau, \hat{z}_{0}, \hat{u}\right), \quad(\text { Fig.3) }
$$

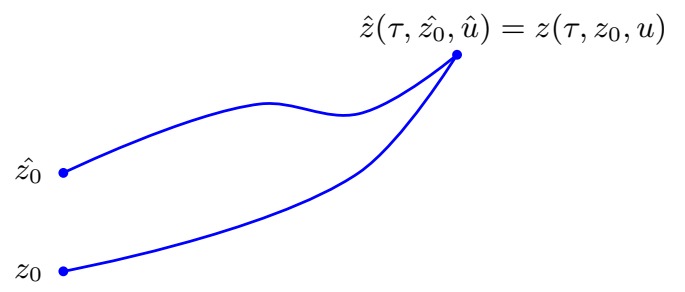

Fig. 3

Definition 1.3 (Null Controllability) The system (1.1) is said to be null controllable on $[0, \tau]$ if for every $z_{0} \in Z=U=L_{2}(\Omega)$ there exists $C([0, \tau] ; U)$ such that the mild solution $z(t)$ of (1.1) corresponding to u verifies:

$$
z(0)=z_{0} \quad \text { and } \quad z(\tau)=0, \quad(\text { Fig.4 })
$$




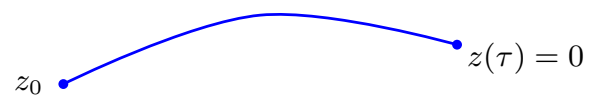

Fig.4

Remark 1.1 It is clear that exact controllability of the system(1.1) implies approximate controllability, null controllability and controllability to trajectories of the system.But, it is well known ([2]) that due to the diffusion effect or the compactness of the semigroup generated by $-\Delta$, the heat equation can never be exactly controllable. We observe also that in the linear case controllability to trajectories and null controllability are equivalent. Nevertheless, the approximate controllability and the null controllability are in general independent. Therefore, in this paper we will be concentrated only on the study of the approximate controllability of the system(1.1).

Recently the interior controllability of the semilinear heat equation (1.1) without impulses has been proved in [13], [14] and [15] under the following condition:

$$
\sup _{(t, z, u) \in Q_{\tau}}|f(t, z, u)-a z-c u|<\infty
$$

where $a, c \in \mathbb{R}$, with $c \neq-1$ and $Q_{\tau}=[0, \tau] \times \mathbb{R} \times \mathbb{R}$.

More recently, in [14], the approximate controllability of the semilinear heat equation (1.1) without impulses has been proved under the following non linear perturbation:

$$
|f(t, z, u)-a z| \leq c|u|^{\beta}+b, \quad \forall u, z \in \mathbb{R}, \quad|u|,|z| \geq R,
$$

where $a, b, c \in \mathbb{R}, R>0$ and $\frac{1}{2} \leq \beta<1$. We note that, the interior approximate controllability of the linear heat equation

$$
\left\{\begin{array}{l}
z_{t}(t, x)=\Delta z(t, x)+1_{\omega} u(t, x) \quad \text { in } \quad(0, \tau] \times \Omega, \\
z=0, \\
z(0, x)=z_{0}(x),
\end{array}\right.
$$

has been study by several authors, particularly by [22],[23],[24]; and in a general fashion in [12].

The controllability of Impulsive Evolution Equations has been studied recently for several authors, but most them study the exact controllability only, to mention: D.N. Chalishajar([4]), studied the exact controllability of impulsive partial neutral functional differential equations with infinite delay, B. Radhakrishnan and K. Balachandran([19]) studied the exact controllability of semilinear impulsive integrodifferential evolution systems with nonlocal conditions and S. Selvi, M. Mallika Arjunan([20]) studied the exact controllability for impulsive differential systems with finite delay. To our knowledge, there are a few works on approximate controllability of impulsive semilinear evolution equations, to mention: Lizhen Chen and Gang $\operatorname{Li}([5])$ 
studied the Approximate controllability of impulsive differential equations with nonlocal conditions, using measure of noncompactness and Monch fixed point theorem, and assuming that the nonlinear term $f(t, z)$ does not depend on the control variable.

Finally, the approximate controllability of the system (1.1) follows from the approximate controllability of (1.7), the compactness of the semigroup generated by the Laplacian operator $-\Delta$, the conditions (1.2) and (1.5) satisfied by the nonlinear term $f, I_{k}$ and the following results:

Proposition 1.1 Let $(X, \Sigma, \mu)$ be a measure space with $\mu(X)<\infty$ and $1 \leq q<r<$ $\infty$. Then $L_{r}(\mu) \subset L_{q}(\mu)$ and

$$
\|f\|_{q} \leq \mu(X)^{\frac{r-q}{r q}}\|f\|_{r}, \quad f \in L_{r}(\mu) .
$$

Proof The proof of this proposition follows from Theorem I.V.6 from [3] by putting $p=\frac{r}{q}>1$ and considering the relation

$$
\int_{X}\left(|f|^{q}\right)^{p} d \mu=\int_{X}|f|^{r} d \mu, \quad \forall f \in L_{r}(\mu) .
$$

Theorem 1.1 (Rothe's Fixed Theorem, [1],[9], [21]) Let E be a Banach space. Let $B \subset E$ be a closed convex subset such that the zero of $E$ is contained in the interior of $B$. Let $\Phi: B \rightarrow E$ be a continuous mapping with $\Phi(B)$ relatively compact in $E$ and $\Phi(\partial B) \subset B$. Then there is a point $x^{*} \in B$ such that $\Phi\left(x^{*}\right)=x^{*}$.

The technique we use here to prove the approximate controllability of the linear part of equation (1.7) is based on the classical Unique Continuation for Elliptic Equations (see [18]) and the following lemma:

Lemma 1.1 (see Lemma 3.14 from [6], pg. 62) Let $\left\{\alpha_{j}\right\}_{j \geq 1}$ and $\left\{\beta_{i, j}: i=\right.$ $1,2, \ldots, m\}_{j \geq 1}$ be two sequences of real numbers such that: $\alpha_{1}>\alpha_{2}>\alpha_{3} \ldots$. Then

$$
\sum_{j=1}^{\infty} e^{\alpha_{j} t} \beta_{i, j}=0, \quad \forall t \in[0, \tau], \quad i=1,2, \cdots, m
$$

iff

$$
\beta_{i, j}=0, \quad i=1,2, \cdots, m ; j=1,2, \cdots, \infty .
$$

\section{Abstract Formulation of the Problem}

In this section we choose a Hilbert space where system (1.1) can be written as an abstract differential equation; to this end, we consider the following results appearing in [6] pg.46, [8] pg.335 and [10] pg.147:

Let us consider the Hilbert space $Z=L_{2}(\Omega)$ and $0<\lambda_{1}<\lambda_{2}<\ldots<\lambda_{j} \longrightarrow \infty$ the eigenvalues of $-\Delta$ with the Dirichlet homogeneous conditions, each one with finite 
multiplicity $\gamma_{j}$ equal to the dimension of the corresponding eigenspace. Then we have the following well known properties

(i) There exists a complete orthonormal set $\left\{\phi_{j, k}\right\}$ of eigenvectors of $A=-\Delta$.

(ii) For all $z \in D(A)$ we have

$$
A z=\sum_{j=1}^{\infty} \lambda_{j} \sum_{k=1}^{\gamma_{j}}<z, \phi_{j, k}>\phi_{j, k}=\sum_{j=1}^{\infty} \lambda_{j} E_{j} z
$$

where $\langle\cdot, \cdot\rangle$ is the inner product in $Z$ and

$$
E_{j} z=\sum_{k=1}^{\gamma_{j}}<z, \phi_{j, k}>\phi_{j, k}
$$

So, $\left\{E_{j}\right\}$ is a family of complete orthogonal projections in $Z$ and $z=\sum_{j=1}^{\infty} E_{j} z, \quad z \in$ $Z$.

(iii) $-A$ generates an analytic semigroup $\{T(t)\}$ given by

$$
T(t) z=\sum_{j=1}^{\infty} e^{-\lambda_{j} t} E_{j} z \text { and }\|T(t)\| \leq e^{-\lambda_{1} t}, \quad t \geq 0 .
$$

Consequently, system (1.1) can be written as an abstract impulsive differential equations in $Z$ :

$$
\begin{cases}z^{\prime}=-A z+B_{\omega} u+f^{e}(t, z, u), & t \in(0, \tau], t \neq t_{k}, \quad z \in Z \\ z(0)=z_{0} & \\ z\left(t_{k}^{+}\right)=z\left(t_{k}^{-}\right)+I_{k}^{e}\left(t_{k}, z\left(t_{k}\right), u\left(t_{k}\right)\right), & k=1,2,3, \ldots, p\end{cases}
$$

where $u \in C([0, \tau] ; U), U=Z, B_{\omega}: U \longrightarrow Z, B_{\omega} u=1_{\omega} u$ is a bounded linear operator, $I_{k}^{e}, f^{e}:[0, \tau] \times Z \times U \rightarrow Z$, are defined by

$I_{k}^{e}(t, z, u)(x)=I_{k}(t, z(x), u(x)), \quad f^{e}(t, z, u)(x)=f(t, z(x), u(x)), \forall x \in \Omega, k=1,2, \ldots, p$.

On the other hand, from conditions (1.2) and (1.5) we get the following estimates.

Proposition 2.1 Under the conditions (1.2)-(1.5) the functions $f^{e}, I_{k}^{e}:[0, \tau] \times Z \times$ $U \rightarrow Z, k=1,2,3, \ldots, p$, defined above satisfy $\forall u, z \in Z=L_{2}(\Omega)$ :

$$
\begin{aligned}
\left\|f^{e}(t, z, u)\right\|_{Z} & \leq \tilde{a}_{0}\|z\|_{Z}^{\alpha_{0}}+\tilde{b}_{0}\|u\|_{Z}^{\beta_{0}}+\tilde{c}_{0} \\
\left\|I_{k}^{e}(t, z, u)\right\|_{Z} & \leq \tilde{a}_{k}\|z\|_{Z}^{\alpha_{k}}+\tilde{b}_{k}\|u\|_{Z}^{\beta_{k}}+\tilde{c}_{k}, \quad k=1,2,3, \ldots, p .
\end{aligned}
$$

Proof.

$$
\begin{aligned}
\left\|f^{e}(t, z, u)\right\|_{Z}^{2} & =\int_{\Omega}|f(t, z(x), u(x))|^{2} d x \\
& \leq \int_{\Omega}\left(a_{0}|z(x)|^{\alpha_{0}}+b_{0}|u(x)|^{\beta_{0}}+c_{0}\right)^{2} d x \\
& \leq \int_{\Omega}\left(4 a_{0}^{2}|z(x)|^{2 \alpha_{0}}+4^{2} b_{0}^{2}|u(x)|^{\beta_{0}}+4^{2} c_{0}^{2}\right) d x \\
& \leq 4 a_{0}^{2} \int_{\Omega}|z(x)|^{2 \alpha_{0}} d x+4^{2} b_{0}^{2} \int_{\Omega}|u(x)|^{2 \beta_{0}} d x+4^{2} c_{0}^{2} \mu(\Omega) .
\end{aligned}
$$


Then

$$
\begin{aligned}
\left\|f^{e}(t, z, u)\right\|_{Z} & \leq 2 a_{0}\left(\int_{\Omega}|z(x)|^{2 \alpha_{0}} d x\right)^{\frac{1}{2}}+4 b_{0}\left(\int_{\Omega}|u(x)|^{2 \beta_{0}} d x\right)^{\frac{1}{2}}+4 c_{0} \sqrt{\mu(\Omega)} \\
& =2 a_{0}\|z\|_{L_{2 \alpha_{0}}}^{\alpha_{0}}+4 b_{0}\|z\|_{L_{2 \beta_{0}}}^{\beta_{0}}+4 c_{0} \sqrt{\mu(\Omega)}
\end{aligned}
$$

Now, since $\frac{1}{2} \leq \alpha_{0}<1 \Leftrightarrow 1 \leq 2 \alpha_{0}<2$ and $\frac{1}{2} \leq \beta_{0}<1 \Leftrightarrow 1 \leq 2 \beta_{0}<2$ applying proposition 1.1, we obtain that:

$$
\left\|f^{e}(t, z, u)\right\|_{Z} \leq 2 a_{0} \mu(\Omega)^{\frac{1-\alpha_{0}}{\alpha_{0}}}\|z\|_{Z}^{\alpha_{0}}+2 b_{0} \mu(\Omega)^{\frac{1-\beta_{0}}{\beta_{0}}}\|u\|_{Z}^{\beta_{0}}+4 c_{0} \sqrt{\mu(\Omega)} .
$$

Analogously, we obtain the following estimate for $k=1,2,3, \ldots, p$

$$
\left\|I_{k}^{e}(t, z, u)\right\|_{Z} \leq 2 a_{k} \mu(\Omega)^{\frac{1-\alpha_{k}}{\alpha_{k}}}\|z\|_{Z}^{\alpha_{k}}+2 b_{k} \mu(\Omega)^{\frac{1-\beta_{k}}{\beta_{k}}}\|u\|_{Z}^{\beta_{k}}+4 c_{k} \sqrt{\mu(\Omega)},
$$

which completes the proof.

\section{Controllability of the Linear Equation without Im- pulses}

In this section we shall present some characterization of the interior approximate controllability of the linear heat equations without impulses. To this end, we note that, for all $z_{0} \in Z$ and $u \in L_{2}(0, \tau ; U)$ the initial value problem

$$
\left\{\begin{array}{l}
z^{\prime}=-A z+B_{\omega} u(t), \quad z \in Z \\
z(0)=z_{0}
\end{array}\right.
$$

where the control function $u$ belongs to $L_{2}(0, \tau ; U)$, admits only one mild solution given by

$$
z(t)=T(t) z_{0}+\int_{0}^{t} T(t-s) B_{\omega} u(s) d s, \quad t \in[0, \tau] .
$$

Definition 3.1 For system (3.1) we define the following concept: The controllability map (for $\tau>0$ ) $G: L_{2}(0, \tau ; U) \longrightarrow Z$ is given by

$$
G u=\int_{0}^{\tau} T(\tau-s) B_{\omega} u(s) d s .
$$

whose adjoint operator $G^{*}: Z \longrightarrow L_{2}(0, \tau ; Z)$ is given by

$$
\left(G^{*} z\right)(s)=B_{\omega}^{*} T^{*}(\tau-s) z, \quad \forall s \in[0, \tau], \quad \forall z \in Z .
$$

Therefore, the Grammian operator $\mathcal{W}: Z \rightarrow Z$ is given

$$
\mathcal{W} z=G G^{*} z=\int_{0}^{\tau} T(\tau-s) B_{\omega} B_{\omega}^{*} T^{*}(\tau-s) d s .
$$

The following lemma holds in general for a linear bounded operator $G: W \rightarrow Z$ between Hilbert spaces $W$ and $Z$. 
Lemma 3.1 (see [6], [7] and [12]) The equation (3.1) is approximately controllable on $[0, \tau]$ if, and only if, one of the following statements holds:

a) $\overline{\operatorname{Rang}(G)}=Z$.

b) $\operatorname{Ker}\left(G^{*}\right)=\{0\}$.

c) $\left\langle G G^{*} z, z\right\rangle>0, z \neq 0$ in $Z$.

d) $\lim _{\alpha \rightarrow 0^{+}} \alpha\left(\alpha I+G G^{*}\right)^{-1} z=0$.

e) $B_{\omega}^{*} T^{*}(t) z=0, \quad \forall t \in[0, \tau], \quad \Rightarrow z=0$.

f) For all $z \in Z$ we have $G u_{\alpha}=z-\alpha\left(\alpha I+G G^{*}\right)^{-1} z$, where

$$
u_{\alpha}=G^{*}\left(\alpha I+G G^{*}\right)^{-1} z, \quad \alpha \in(0,1] .
$$

So, $\lim _{\alpha \rightarrow 0} G u_{\alpha}=z$ and the error $E_{\alpha} z$ of this approximation is given by

$$
E_{\alpha} z=\alpha\left(\alpha I+G G^{*}\right)^{-1} z, \quad \alpha \in(0,1] .
$$

Remark 3.1 The Lemma 3.1 implies that the family of linear operators $\Gamma_{\alpha}: Z \rightarrow L_{2}(0, \tau ; U)$, defined for $0<\alpha \leq 1$ by

$$
\Gamma_{\alpha} z=B_{\omega}^{*} T^{*}(\cdot)\left(\alpha I+G G^{*}\right)^{-1} z=G^{*}\left(\alpha I+G G^{*}\right)^{-1} z,
$$

is an approximate inverse for the right of the operator $G$ in the sense that

$$
\lim _{\alpha \rightarrow 0} G \Gamma_{\alpha}=I
$$

in the strong topology.

Proposition 3.1 (See [15]) If $\overline{\operatorname{Rang}(G)}=Z$, then

$$
\sup _{\alpha>0}\left\|\alpha\left(\alpha I+G G^{*}\right)^{-1}\right\| \leq 1
$$

Remark 3.2 The proof of the following theorem follows from foregoing characterization of dense range linear operators and the classical Unique Continuation for Elliptic Equations (see [18]), and it is similar to the one given in Theorem 4.1 in [14].

Theorem 3.1 System (3.1) is approximately controllable on $[0, \tau]$. Moreover, a sequence of controls steering the system (3.1) from initial state $z_{0}$ to an $\epsilon$ neighborhood of the final state $z_{1}$ at time $\tau>0$ is given by

$$
u_{\alpha}(t)=B_{\omega}^{*} T^{*}(\tau-t)\left(\alpha I+G G^{*}\right)^{-1}\left(z_{1}-T(\tau) z_{0}\right),
$$

and the error of this approximation $E_{\alpha}$ is given by

$$
E_{\alpha}=\alpha\left(\alpha I+G G^{*}\right)^{-1}\left(z_{1}-T(\tau) z_{0}\right) .
$$


Proof . It is enough to show that $\overline{\operatorname{Rang}(G)}=Z$ or $\operatorname{Ker}\left(G^{*}\right)=\{0\}$. To this end, we observe that $B_{\omega}=B_{\omega}^{*}$ and $T^{*}(t)=T(t)$. Suppose that

$$
B_{\omega}^{*} T^{*}(t) z=0, \quad \forall t \in[0, \tau] .
$$

Then,

$$
\begin{aligned}
B_{\omega}^{*} T^{*}(t) z & =\sum_{j=1}^{\infty} e^{-\lambda_{j} t} B_{\omega}^{*} E_{j} z=\sum_{j=1}^{\infty} e^{-\lambda_{j} t} \sum_{k=1}^{\gamma_{j}}<z, \phi_{j, k}>1_{\omega} \phi_{j, k}=0 . \\
& \Longleftrightarrow \sum_{j=1}^{\infty} e^{-\lambda_{j} t} \sum_{k=1}^{\gamma_{j}}<z, \phi_{j, k}>1_{\omega} \phi_{j, k}(x)=0, \quad \forall x \in \omega .
\end{aligned}
$$

Hence, from Lemma 1.1, we obtain that

$$
E_{j} z(x)=\sum_{k=1}^{\gamma_{j}}<z, \phi_{j, k}>\phi_{j, k}(x)=0, \quad \forall x \in \omega, \quad j=1,2,3, \ldots
$$

Now, putting $f(x)=\sum_{k=1}^{\gamma_{j}}<z, \phi_{j, k}>\phi_{j, k}(x), \quad \forall x \in \Omega$, we obtain that

$$
\left\{\begin{array}{l}
\left(\Delta+\lambda_{j} I\right) f \equiv 0 \quad \text { in } \Omega, \\
f(x)=0 \quad \forall x \in \omega .
\end{array}\right.
$$

Then, from the classical Unique Continuation for Elliptic Equations (see [18]), it follows that $f(x)=0, \quad \forall x \in \Omega$. So,

$$
\sum_{k=1}^{\gamma_{j}}<z, \phi_{j, k}>\phi_{j, k}(x)=0, \quad \forall x \in \Omega .
$$

On the other hand, $\left\{\phi_{j, k}\right\}$ is a complete orthonormal set in $Z=L_{2}(\Omega)$, which implies that $\left\langle z, \phi_{j, k}\right\rangle=0$.

Therefore, $E_{j} z=0, \quad j=1,2,3, \ldots$, which implies that $z=0$. So, $\overline{\operatorname{Rang}(G)}=Z$. Hence, the system (3.1) is approximately controllable on $[0, \tau]$, and the remainder of the proof follows from Lemma 3.1 .

Lemma 3.2 Let $S$ be any dense subspace of $L_{2}(0, \tau ; U)$. Then, system (3.1) is approximately controllable with control $u \in L_{2}(0, \tau ; U)$ if, and only if, it is approximately controllable with control $u \in S$. i.e.,

$$
\overline{\operatorname{Rang}(G)}=Z \Longleftrightarrow \overline{\operatorname{Rang}\left(\left.G\right|_{S}\right)}=Z,
$$

where $\left.G\right|_{S}$ is the restriction of $G$ to $S$.

Proof $(\Rightarrow)$ Suppose $\overline{\operatorname{Rang}(G)}=Z$ and $\bar{S}=L_{2}(0, \tau ; U)$. Then, for a given $\epsilon>0$ and $z \in Z$ there exits $u \in L_{2}(0, \tau ; U)$ and a sequence $\left\{u_{n}\right\}_{n \geq 1} \subset S$ such that

$$
\|G u-z\|<\frac{\epsilon}{2} \text { and } \lim _{n \rightarrow \infty} u_{n}=u \text {. }
$$

Therefore, $\lim _{n \rightarrow \infty} G u_{n}=G u$ and $\left\|G u_{n}-z\right\|<\epsilon$ for $n$ big enough. Hence, $\overline{\operatorname{Rang}\left(\left.G\right|_{S}\right)}=Z$.

$(\Leftarrow)$ This side is trivial. 
Remark 3.3 According to the previous lemma, if the system is controllable, it is approximately controllable with control functions in the following dense spaces of $L_{2}(0, \tau ; U)$ :

$$
S=C([0, \tau] ; U), \quad S=C^{\infty}(0, \tau ; U), \quad S=P C(J) .
$$

Moreover, the operators $G, \mathcal{W}$ and $\Gamma$ are well define in the space of continuous functions: $G: C([0, \tau] ; U) \longrightarrow Z$ by

$$
G u=\int_{0}^{\tau} T(\tau-s) B_{\omega} u(s) d s,
$$

and $G^{*}: Z \longrightarrow C([0, \tau] ; U)$ by

$$
\left(G^{*} z\right)(s)=B^{*}(s) T^{*}(\tau-s) z, \quad \forall s \in[0, \tau] . \quad \forall z \in Z .
$$

Also, the Controllability Grammian operator still the same $\mathcal{W}: Z \rightarrow Z$

$$
\mathcal{W} z=G G^{*} z=\int_{0}^{\tau} T(\tau-s) B_{\omega} B_{\omega}^{*}(s) T^{*}(\tau-s) z d s .
$$

Finally, the operators $\Gamma_{\alpha}: Z \rightarrow C([0, \tau] ; U)$ defined for $0<\alpha \leq 1$ by

$$
\Gamma_{\alpha} z=B_{\omega}^{*} T^{*}(\tau-\cdot)(\alpha I+\mathcal{W})^{-1} z=G^{*}\left(\alpha I+G G^{*}\right)^{-1} z
$$

is an approximate inverse for the right of the operator $G$ in the sense that

$$
\lim _{\alpha \rightarrow 0} G \Gamma_{\alpha}=I \text {. }
$$

\section{Controllability of the Semilinear System}

In this section we shall prove the main result of this paper, the interior approximate controllability of the Semilinear Impulsive Heat Equation given by (1.1), which is equivalent to prove the approximate controllability of the system (2.4). To this end, for all $z_{0} \in Z$ and $u \in C([0, \tau] ; U)$ the initial value problem

$$
\begin{cases}z^{\prime}=-A z+B_{\omega} u+f^{e}(t, z, u), & t \in(0, \tau], t \neq t_{k}, \quad z \in Z \\ z(0)=z_{0}, & \\ z\left(t_{k}^{+}\right)=z\left(t_{k}^{-}\right)+I_{k}^{e}\left(t, z\left(t_{k}\right), u\left(t_{k}\right)\right), & k=1,2,3, \ldots, p .\end{cases}
$$

admits only one mild solution given by

$$
\begin{aligned}
z_{u}(t) & =T(t) z_{0}+\int_{0}^{t} T(t-s) B_{\omega} u(s) d s \\
& +\int_{0}^{t} T(t-s) f^{e}\left(s, z_{u}(s), u(s)\right) d s \\
& +\sum_{0<t_{k}<t} T\left(t-t_{k}\right) I_{k}^{e}\left(t_{k}, z\left(t_{k}\right), u\left(t_{k}\right)\right), \quad t \in[0, \tau] .
\end{aligned}
$$


Now, we are ready to present and prove the main result of this paper, which is the interior approximate controllability of the semilinear impulsive heat equation (1.1). We shall define the operator $\mathcal{K}^{\alpha}: P C([0, \tau] ; Z) \times C([0, \tau] ; U) \rightarrow P C([0, \tau] ; Z) \times$ $C([0, \tau] ; U)$ by the following formula:

$$
(y, v)=\left(\mathcal{K}_{1}^{\alpha}(z, u), \mathcal{K}_{2}^{\alpha}(z, u)\right)=\mathcal{K}^{\alpha}(z, u)
$$

where

$$
\begin{aligned}
y(t) & =\mathcal{K}_{1}^{\alpha}(z, u)(t)=T(t) z_{0}+\int_{0}^{t} T(t-s) B_{\omega}\left(\Gamma_{\alpha} \mathcal{L}(z, u)\right)(s) d s \\
& +\int_{0}^{t} T(t-s) f^{e}(s, z(s), u(s)) d s+\sum_{0<t_{k}<t} T\left(t-t_{k}\right) I_{k}^{e}\left(t_{k}, z\left(t_{k}\right), u\left(t_{k}\right)\right),
\end{aligned}
$$

and

$$
v(t)=\mathcal{K}_{2}^{\alpha}(z, u)(t)=\left(\Gamma_{\alpha} \mathcal{L}(z, u)\right)(t)=B_{\omega}^{*} T^{*}(\tau-t)(\alpha I+\mathcal{W})^{-1} \mathcal{L}(z, u),
$$

with $\mathcal{L}: P C([0, \tau] ; Z) \times C([0, \tau] ; U) \rightarrow Z$ is given by

$$
\begin{aligned}
\mathcal{L}(z, u) & =z_{1}-T(\tau) z_{0}-\int_{0}^{\tau} T(\tau-s) f^{e}(s, z(s), u(s)) d s \\
& -\sum_{0<t_{k}<\tau} T\left(\tau-t_{k}\right) I_{k}^{e}\left(t_{k}, z\left(t_{k}\right), u\left(t_{k}\right)\right) .
\end{aligned}
$$

Theorem 4.1 The nonlinear system (1.1) is approximately controllable on $[0, \tau]$. Moreover, a sequence of controls steering the system (1.1) from initial state $z_{0}$ to an $\epsilon$-neighborhood of the final state $z_{1}$ at time $\tau>0$ is given by

$$
u_{\alpha}(t)=B_{\omega}^{*} T^{*}(\tau-t)(\alpha I+\mathcal{W})^{-1} \mathcal{L}\left(z_{\alpha}, u_{\alpha}\right),
$$

and the error of this approximation $E_{\alpha} z$ is given by

$$
E_{\alpha} z=\alpha(\alpha I+\mathcal{W})^{-1} \mathcal{L}\left(z_{\alpha}, u_{\alpha}\right)
$$

where

$$
\begin{aligned}
z_{\alpha}(t) & =T(t) z_{0}+\int_{0}^{t} T(t-s) B_{\omega} u_{\alpha}(s) d s \\
& +\int_{0}^{t} T(t-s) f^{e}\left(s, z_{\alpha}(s), u_{\alpha}(s)\right) d s \\
& +\sum_{0<t_{k}<t} T\left(t-t_{k}\right) I_{k}^{e}\left(t_{k}, z_{\alpha}\left(t_{k}\right), u_{\alpha}\left(t_{k}\right)\right), \quad t \in[0, \tau] .
\end{aligned}
$$

Proof We shall prove this Theorem by claims. Before we note that $\left\|B_{\omega}\right\|=1$ and $\|T(t)\| \leq e^{-\lambda_{1} t}, \quad t \geq 0$. 
Claim 1. The operator $\mathcal{K}^{\alpha}$ is continuous. In fact, it is enough to prove that the operators:

$$
\mathcal{K}_{1}^{\alpha}: P C([0, \tau] ; Z) \times C([0, \tau] ; U) \rightarrow P C([0, \tau] ; Z)
$$

and

$$
\mathcal{K}_{2}^{\alpha}: P C([0, \tau] ; Z) \times C([0, \tau] ; U) \rightarrow C([0, \tau] ; U),
$$

define above are continuous. The continuity of $\mathcal{K}_{1}^{\alpha}$ follows from the continuity of the nonlinear functions $f^{\alpha}(t, z, u), I_{k}^{e}(t, z, u)$ and the following estimate

$$
\begin{aligned}
\| \mathcal{K}_{1}^{\alpha}(z, u)(t) & -\mathcal{K}_{1}^{\alpha}(w, v)(t)\left\|\leq \int_{0}^{t} e^{-\lambda_{1}(t-s)}\right\|(\alpha I+\mathcal{W})^{-1}\|\| \mathcal{L}(z, u)-\mathcal{L}(w, v) \| d s \\
& +\int_{0}^{t} e^{-\lambda_{1}(t-s)}\left\|f^{e}(s, z(s), u(s))-f^{e}(s, w(s), v(s))\right\| d s \\
& +\sum_{0<t_{k}<t} e^{-\lambda_{1}\left(t-t_{k}\right)}\left\|I_{k}^{e}\left(t_{k}, z\left(t_{k}\right), u\left(t_{k}\right)\right)-I_{k}^{e}\left(t_{k}, w\left(t_{k}\right), v\left(t_{k}\right)\right)\right\| .
\end{aligned}
$$

On the other hand,

$$
\begin{aligned}
\|\mathcal{L}(z, u)-\mathcal{L}(w, v)\| & \leq \tau \sup _{s \in[0, \tau]}\left\|f^{e}(s, z(s), u(s))-f^{e}(s, w(s), v(s))\right\| \\
& +\sum_{0<t_{k}<\tau} e^{-\lambda_{1}\left(t-t_{k}\right)}\left\|I_{k}^{e}\left(t_{k}, z\left(t_{k}\right), u\left(t_{k}\right)\right)-I_{k}^{e}\left(t_{k}, w\left(t_{k}\right), v\left(t_{k}\right)\right)\right\| .
\end{aligned}
$$

Therefore,

$$
\begin{aligned}
\left\|\mathcal{K}_{1}^{\alpha}(z, u)-\mathcal{K}_{1}^{\alpha}(w, v)\right\| & \leq L_{1} \sup _{s \in[0, \tau]}\left\|f^{e}(s, z(s), u(s))-f^{e}(s, w(s), v(s))\right\| \\
& +L_{2} \sum_{0<t_{k}<\tau}\left\|I_{k}^{e}\left(t_{k}, z\left(t_{k}\right), u\left(t_{k}\right)\right)-I_{k}^{e}\left(t_{k}, w\left(t_{k}\right), v\left(t_{k}\right)\right)\right\| .
\end{aligned}
$$

where $L_{1}=\tau\left(\tau\left\|(\alpha I+\mathcal{W})^{-1}\right\|+1\right)$ and $L_{2}=\left(1+\tau\left\|(\alpha I+\mathcal{W})^{-1}\right\|\right)$.

The continuity of the operator $\mathcal{K}_{2}^{\alpha}$ follows from the continuity of the operators $\mathcal{L}$ and $\Gamma_{\alpha}$ define above.

Claim 2. The operator $\mathcal{K}^{\alpha}$ is compact. In fact, let $D$ be a bounded subset of $P C(J ; Z) \times C(J ; U)$. It follows that $\forall(z, u) \in D$, we have

$$
\begin{aligned}
\left\|f^{e}(\cdot, z, u)\right\| & \leq L_{3}, \quad\left\|(\alpha I+\mathcal{W})^{-1} \mathcal{L}(z, u)\right\| \leq L_{4}, \\
\|\mathcal{L}(z, u)\| & \leq L_{5}, \quad\left\|I_{k}^{e}(\cdot, z, u)\right\| \leq l_{k}, \quad k=1,2, \ldots, p .
\end{aligned}
$$

Therefore, $\mathcal{K}(D)$ is uniformly bounded.

Now, consider the following estimate:

$$
\begin{aligned}
\left\|\left|\mathcal{K}^{\alpha}(z, u)\left(t_{2}\right)-\mathcal{K}^{\alpha}(z, u)\left(t_{1}\right) \|\right|\right. & =\left\|\mathcal{K}_{1}^{\alpha}(z, u)\left(t_{2}\right)-\mathcal{K}_{1}^{\alpha}(z, u)\left(t_{1}\right)\right\| \\
& +\left\|\mathcal{K}_{2}^{\alpha}(z, u)\left(t_{2}\right)-\mathcal{K}_{2}^{\alpha}(z, u)\left(t_{1}\right)\right\| .
\end{aligned}
$$


Without lose of generality we assume that $0<t_{1}<t_{2}$. On the other hand we have:

$$
\begin{aligned}
& \left\|\mathcal{K}_{1}^{\alpha}(z, u)\left(t_{2}\right)-\mathcal{K}_{1}^{\alpha}(z, u)\left(t_{1}\right)\right\| \leq\left\|T\left(t_{2}\right)-T\left(t_{1}\right)\right\|\left\|z_{0}\right\| \\
& \quad+\int_{0}^{t_{1}}\left\|T\left(t_{2}-s\right)-T\left(t_{1}-s\right)\right\|\|\mathcal{L}(z, u)(s)\| d s \\
& +\int_{t_{1}}^{t_{2}}\left\|T\left(t_{2}-s\right)\right\|\|\mathcal{L}(z, u)(s)\| d s \\
& +\int_{0}^{t_{1}}\left\|T\left(t_{2}-s\right)-T\left(t_{1}-s\right)\right\|\left\|f^{e}(s, z(s), u(s))\right\| d s \\
& +\int_{t_{1}}^{t_{2}}\left\|T\left(t_{2}-s\right)\right\|\left\|f^{e}(s, z(s), u(s))\right\| d s \\
& +\sum_{0<t_{k}<t_{1}}\left\|T\left(t_{2}-t_{k}\right)-T\left(t_{1}-t_{k}\right)\right\|\left\|I_{k}^{e}\left(t_{k}, z\left(t_{k}\right), u\left(t_{k}\right)\right)\right\| \\
& +\sum_{t_{1}<t_{k}<t_{2}}\left\|T\left(t_{2}-t_{k}\right) I_{k}^{e}\left(t_{k}, z\left(t_{k}\right), u\left(t_{k}\right)\right)\right\|,
\end{aligned}
$$

and

$$
\left\|\mathcal{K}_{2}^{\alpha}(z, u)\left(t_{2}\right)-\mathcal{K}_{2}^{\alpha}(z, u)\left(t_{1}\right)\right\| \leq\left\|T^{*}\left(\tau-t_{2}\right)-T^{*}\left(\tau-t_{1}\right)\right\|\left\|(\alpha I+\mathcal{W})^{-1} \mathcal{L}(z, u)\right\| .
$$

On the other hand, since $T(t)$ is a compact operator for $t>0$, then from [17] we know that the function $0<t \rightarrow T(t)$ is uniformly continuous. So,

$$
\lim _{\left|t_{2}-t_{1}\right| \rightarrow 0}\left\|T\left(t_{2}\right)-T\left(t_{1}\right)\right\|=0 .
$$

Consequently, if we take a sequence $\left\{\phi_{j}: j=1,2, \ldots\right\}$ on $\mathcal{K}^{\alpha}(D)$, this sequence is uniformly bounded and equicontinuous on the interval $\left[0, t_{1}\right]$ and, by Arzela theorem, there is a subsequence $\left\{\phi_{j}^{1}: j=1,2, \ldots\right\}$ of $\left\{\phi_{j}: j=1,2, \ldots\right\}$, which is uniformly convergent on $\left[0, t_{1}\right]$.

Consider the sequence $\left\{\phi_{j}^{1}: j=1,2, \ldots\right\}$ on the interval $\left(t_{1}, t_{2}\right]$. On this interval the sequence $\left\{\phi_{j}^{1}: j=1,2, \ldots\right\}$ is uniformly bounded and equicontinuous, and for the same reason, it has a subsequence $\left\{\phi_{j}^{2}\right\}$ uniformly convergent on $\left[0, t_{2}\right]$.

Continuing this process for the intervals $\left(t_{2}, t_{3}\right],\left(t_{3}, t_{4}\right], \ldots,\left(t_{p}, \tau\right]$, we see that the sequence $\left\{\phi_{j}^{p+1}: j=1,2, \ldots\right\}$ converges uniformly on the interval $[0, \tau]$. This means that $\overline{\mathcal{K}^{\alpha}(D)}$ is compact, which implies that the operator $\mathcal{K}^{\alpha}$ is compact.

Claim 3.

$$
\lim _{\||(z, u) \|| \rightarrow \infty} \frac{\left\|\left|\mathcal{K}^{\alpha}(z, u) \|\right|\right.}{\||(z, u) \||}=0
$$

where $\||(z, u)\|\mid=\| z\|+\| u \|$ is the norm in the space $P C([0, \tau] ; Z) \times C(0, \tau ; Z)$. In fact, consider the following estimates:

$$
\|\mathcal{L}(z, u)\| \leq M_{1}+M_{2}\left\{\bar{a}_{0}\|z\|^{\alpha_{0}}+\bar{b}_{0}\|u\|^{\beta_{0}}+\bar{c}_{0}\right\}+M_{3} \sum_{0<t_{k}<\tau}\left\{\bar{a}_{k}\|z\|^{\alpha_{k}}+\bar{b}_{k}\|u\|^{\beta_{k}}+\bar{c}_{k}\right\},
$$


where

$$
\begin{array}{rr}
M_{1} & =\left\|z_{1}\right\|+e^{-\lambda_{1} \tau}\left\|z_{0}\right\|, \quad M_{2}=\frac{1}{-\lambda_{1}}\left(e^{-\lambda_{1} \tau}-1\right) \quad \text { and } M_{3}=e^{-\lambda_{1} \tau} . \\
\left\|\mathcal{K}_{2}^{\alpha}(z, u)\right\| & \leq M_{3} M_{1}\left\|(\alpha I+\mathcal{W})^{-1}\right\|+M_{3} M_{2}\left\|(\alpha I+\mathcal{W})^{-1}\right\|\left\{\bar{a}_{0}\|z\|^{\alpha_{0}}+\bar{b}_{0}\|u\|^{\beta_{0}}+\bar{c}_{0}\right\} \\
+ & M_{3} M_{2}\left\|(\alpha I+\mathcal{W})^{-1}\right\| \sum_{0<t_{k}<\tau}\left\{\bar{a}_{k}\|z\|^{\alpha_{k}}+\bar{b}_{k}\|u\|^{\beta_{k}}+\bar{c}_{k}\right\} .
\end{array}
$$

and

$$
\begin{aligned}
\left\|\mathcal{K}_{1}^{\alpha}(z, u)\right\| & \leq M_{3}\left\{\left\|z_{0}\right\|+M_{1} M_{2}\left\|(\alpha I+\mathcal{W})^{-1}\right\|\right\} \\
& +M_{2}\left\{1+M_{2} M_{3}\left\|(\alpha I+\mathcal{W})^{-1}\right\|\right\}\left\{\bar{a}_{0}\|z\|^{\alpha_{0}}+\bar{b}_{0}\|u\|^{\beta_{0}}+c_{0}\right\} \\
& +M_{3}\left\{1+M_{2} M_{3}\left\|(\alpha I+\mathcal{W})^{-1}\right\|\right\} \sum_{0<t_{k}<\tau}\left\{\bar{a}_{k}\|z\|^{\alpha_{k}}+\bar{b}_{k}\|u\|^{\beta_{k}}+\bar{c}_{k}\right\} .
\end{aligned}
$$

Therefore,

$$
\begin{aligned}
\left\|\mid \mathcal{K}^{\alpha}(z, u)\right\| & =\left\|\mathcal{K}_{1}^{\alpha}(z, u)\right\|+\left\|\mathcal{K}_{2}^{\alpha}(z, u)\right\| \leq M_{4} \\
& +\left\{M_{3} M_{2}\left\|(\alpha I+\mathcal{W})^{-1}\right\|\left\{1+2 M_{2}\right\}\left\{\bar{a}_{0}\|z\|^{\alpha_{0}}+\bar{b}_{0}\|u\|^{\beta_{0}}+\bar{c}_{0}\right\}\right. \\
& +\left\{M_{3} M_{2}\left\|(\alpha I+\mathcal{W})^{-1}\right\|\left\{1+M_{3}\right\}+M_{3}\right\} \sum_{0<t_{k}<\tau}\left\{\bar{a}_{k}\|z\|^{\alpha_{k}}+\bar{b}_{k}\|u\|^{\beta_{k}}+\bar{c}_{k}\right\},
\end{aligned}
$$

where $M_{4}$ is given by:

$$
M_{4}=M_{3}\left\{\left\|z_{0}\right\|+\left(M_{2}+1\right) M_{1}\left\|(\alpha I+\mathcal{W})^{-1}\right\|\right\} .
$$

Hence

$$
\begin{aligned}
\frac{\left\|\mathcal{K}^{\alpha}(z, u)\right\| \mid}{\||(z, u) \||} \leq & \frac{M_{4}}{\|z\|+\|u\|} \\
+ & \left\{M_{3} M_{2}\left\|(\alpha I+\mathcal{W})^{-1}\right\|\left\{1+M_{2}\right\}\right\} \\
& \times\left\{\bar{a}_{0}\|z\|^{\alpha_{0}-1}+\bar{b}_{0}\|u\|^{\beta_{0}-1}+\frac{\bar{c}_{0}}{\|z\|+\|u\|}\right\} \\
+ & \left\{M_{3} M_{2}\left\|(\alpha I+\mathcal{W})^{-1}\right\|\left\{1+M_{3}\right\}+M_{3}\right\} \times \\
& \sum_{0<t_{k}<\tau}\left\{\bar{a}_{k}\|z\|^{\alpha_{k}-1}+\bar{b}_{k}\|u\|^{\beta_{k}-1}+\frac{\bar{c}_{k}}{\|z\|+\|u\|}\right\},
\end{aligned}
$$

and

$$
\lim _{\||(z, u) \|| \rightarrow \infty} \frac{\left\|\left|\mathcal{K}^{\alpha}(z, u) \|\right|\right.}{\||(z, u) \||}=0 .
$$

Claim 4. The operator $\mathcal{K}^{\alpha}$ has a fixed point. In fact, for a fixed $0<\rho<1$, there exists $R>0$ big enough such that

$$
\left\|\left|\mathcal{K}^{\alpha}(z, u)\||\leq \rho\||(z, u)\||, \quad\||(z, u) \||=R .\right.\right.
$$


Hence, if we denote by $B(0, R)$ the ball of center zero and radius $R>0$, we get that $\mathcal{K}^{\alpha}(\partial B(0, R)) \subset B(0, R)$. Since $\mathcal{K}^{\alpha}$ is compact and maps the sphere $\partial B(0, R)$ into the interior of the ball $B(0, R)$, we can apply Rothe's fixed point Theorem 1.1 to ensure the existence of a fixed point $\left(z_{\alpha}, u_{\alpha}\right) \in B(0, R) \subset P C([0, \tau] ; Z) \times C([0, \tau] ; U)$ such that

$$
\left(z_{\alpha}, u_{\alpha}\right)=\mathcal{K}^{\alpha}\left(z_{\alpha}, u_{\alpha}\right) .
$$

Claim 5. The sequence $\left\{\left(z_{\alpha}, u_{\alpha}\right)\right\}_{\alpha \in(0,1]}$ is bounded. In fact, for the purpose of contradiction, let us assume that $\left\{\left(z_{\alpha}, u_{\alpha}\right)\right\}_{\alpha \in(0,1]}$ is unbounded. Then, there exits a subsequence $\left\{\left(z_{\alpha_{n}}, u_{\alpha_{n}}\right)\right\}_{\alpha \in(0,1]} \subset\left\{\left(z_{\alpha}, u_{\alpha}\right)\right\}_{\alpha \in(0,1]}$ such that

$$
\lim _{n \rightarrow \infty}\left\|\left|\left(z_{\alpha_{n}}, u_{\alpha_{n}}\right) \|\right|=\infty .\right.
$$

On the other hand, from (4.7) we know for all $\alpha \in(0,1]$ that

$$
\lim _{n \rightarrow \infty} \frac{\left\|\left|\mathcal{K}^{\alpha}\left(z_{\alpha_{n}}, u_{\alpha_{n}}\right) \|\right|\right.}{\left\|\left|\left(z_{\alpha_{n}}, u_{\alpha_{n}}\right) \|\right|\right.}=0 .
$$

Particularly, we have the following situation:
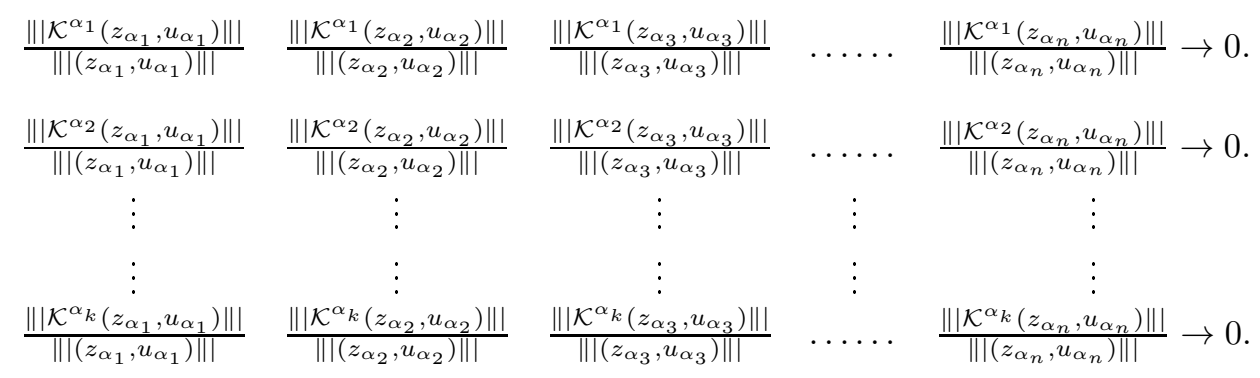

Now, applying Cantor's diagonalization process, we obtain that

$$
\lim _{n \rightarrow \infty} \frac{\left\|\left|\mathcal{K}^{\alpha_{n}}\left(z_{\alpha_{n}}, u_{\alpha_{n}}\right) \|\right|\right.}{\left\|\left|\left(z_{\alpha_{n}}, u_{\alpha_{n}}\right) \|\right|\right.}=0,
$$

and from (4.8) we have that

$$
\frac{\left\|\left|\mathcal{K}^{\alpha_{n}}\left(z_{\alpha_{n}}, u_{\alpha_{n}}\right) \|\right|\right.}{\left\|\left|\left(z_{\alpha_{n}}, u_{\alpha_{n}}\right) \|\right|\right.}=1
$$

which is evidently a contradiction. Then, the claim is true and there exists $\gamma>0$ such that

$$
\left\|\left|\left(z_{\alpha_{n}}, u_{\alpha_{n}}\right) \|\right| \leq \gamma, \quad(0<\alpha \leq 1) .\right.
$$

Therefore, without loss of generality, we can assume that the sequence $\mathcal{L}\left(z_{\alpha}, u_{\alpha}\right)$ converges to $y \in Z$. So, if

$$
u_{\alpha}=\Gamma_{\alpha} \mathcal{L}\left(z_{\alpha}, u_{\alpha}\right)=G^{*}\left(\alpha I+G G^{*}\right)^{-1} \mathcal{L}\left(z_{\alpha}, u_{\alpha}\right) .
$$


Then,

$$
\begin{aligned}
G u_{\alpha} & =G \Gamma_{\alpha} \mathcal{L}\left(z_{\alpha}, u_{\alpha}\right)=G G^{*}\left(\alpha I+G G^{*}\right)^{-1} \mathcal{L}\left(z_{\alpha}, u_{\alpha}\right) \\
& =\left(\alpha I+G G^{*}-\alpha I\right)\left(\alpha I+G G^{*}\right)^{-1} \mathcal{L}\left(z_{\alpha}, u_{\alpha}\right) \\
& =\mathcal{L}\left(z_{\alpha}, u_{\alpha}\right)-\alpha\left(\alpha I+G G^{*}\right)^{-1} \mathcal{L}\left(z_{\alpha}, u_{\alpha}\right)
\end{aligned}
$$

Hence,

$$
G u_{\alpha}-\mathcal{L}\left(z_{\alpha}, u_{\alpha}\right)=-\alpha\left(\alpha I+G G^{*}\right)^{-1} \mathcal{L}\left(z_{\alpha}, u_{\alpha}\right) .
$$

To conclude the proof of this Theorem, it enough to prove that

$$
\lim _{\alpha \rightarrow 0}\left\{-\alpha\left(\alpha I+G G^{*}\right)^{-1}\right\} \mathcal{L}\left(z_{\alpha}, u_{\alpha}\right)=0 .
$$

From Lemma 3.1.d) we get that

$$
\begin{aligned}
\lim _{\alpha \rightarrow 0}\left\{\alpha\left(\alpha I+G G^{*}\right)^{-1} \mathcal{L}\left(z_{\alpha}, u_{\alpha}\right)\right\} & =\lim _{\alpha \rightarrow 0} \alpha\left(\alpha I+G G^{*}\right)^{-1} y \\
& +\lim _{\alpha \rightarrow 0} \alpha\left(\alpha I+G G^{*}\right)^{-1}\left(\mathcal{L}\left(z_{\alpha}, u_{\alpha}\right)-y\right) \\
& =\lim _{\alpha \rightarrow 0}-\alpha\left(\alpha I+G G^{*}\right)^{-1}\left(\mathcal{L}\left(z_{\alpha}, u_{\alpha}\right)-y\right)
\end{aligned}
$$

On the other hand, from Proposition 3.1, we get that

$$
\left.\left\|\alpha\left(\alpha I+G G^{*}\right)^{-1}\left(\mathcal{L}\left(z_{\alpha}, u_{\alpha}\right)-y\right)\right\| \leq \| \mathcal{L}\left(z_{\alpha}, u_{\alpha}\right)-y\right) \| .
$$

Therefore, since $\mathcal{L}\left(z_{\alpha}, u_{\alpha}\right)$ converges to $y$, we get that

$$
\lim _{\alpha \rightarrow 0}\left\{-\alpha\left(\alpha I+G G^{*}\right)^{-1}\left(\mathcal{L}\left(z_{\alpha}, u_{\alpha}\right)-y\right)\right\}=0 .
$$

Consequently,

$$
\lim _{\alpha \rightarrow 0}\left\{-\alpha\left(\alpha I+G G^{*}\right)^{-1} \mathcal{L}\left(z_{\alpha}, u_{\alpha}\right)\right\}=0 .
$$

Then,

Therefore,

$$
\lim _{\alpha \rightarrow 0}\left\{G u_{\alpha}-\mathcal{L}\left(z_{\alpha}, u_{\alpha}\right)\right\}=0 .
$$

$$
\begin{aligned}
\lim _{\alpha \rightarrow 0}\left\{T(\tau) z_{0}+\int_{0}^{\tau} T(\tau-s) B_{\omega} u_{\alpha}(s) d s\right. & +\int_{0}^{\tau} T(\tau-s) f^{e}\left(s, z_{\alpha}(s), u_{\alpha}(s)\right) d s \\
& \left.+\sum_{0<t_{k}<\tau} T\left(\tau-t_{k}\right) I_{k}^{e}\left(z_{\alpha}\left(t_{k}\right), u_{\alpha}\left(t_{k}\right)\right)\right\}=z_{1},
\end{aligned}
$$

and the proof of the theorem is completed.

As a consequence of the foregoing theorem we can prove the following characterization:

Theorem 4.2 The Impulsive Semilinear System (1.1) is approximately controllable if for all states $z_{0}$ and a final state $z_{1}$ and $\alpha \in(0,1]$ the operator $\mathcal{K}^{\alpha}$ given by (4.4)-(4.6) has a fixed point and the sequence $\left\{\mathcal{L}\left(z_{\alpha}, u_{\alpha}\right)\right\}_{\alpha \in(0,1]}$ converges. i.e.,

$$
\begin{gathered}
\left(z_{\alpha}, u_{\alpha}\right)=\mathcal{K}^{\alpha}\left(z_{\alpha}, u_{\alpha}\right), \\
\lim _{\alpha \rightarrow 0} \mathcal{L}\left(z_{\alpha}, u_{\alpha}\right)=y \in Z .
\end{gathered}
$$




\section{$5 \quad$ Final Remark}

Our technique is simple and can be apply to those system involving compact semigroups like some control system governed by diffusion processes. For example, the Benjamin -Bona-Mohany Equation, the strongly damped wave equations, beam equations, etc.

Example 5.1 The original Benjamin -Bona-Mohany Equation is a non-linear one, in [16] the authors proved the approximate controllability of the linear part of this equation, which is the fundamental base for the study of the controllability of the non linear BBM equation. So, our next work is concerned with the controllability of non linear BBM equation

$$
\left\{\begin{array}{l}
z_{t}-a \Delta z_{t}-b \Delta z=1_{\omega} u(t, x)+f(t, z, u(t)), \quad t \in(0, \tau), \quad x \in \Omega, \\
z(t, x)=0, \quad t \geq 0, \quad x \in \partial \Omega \\
z(0, x)=z_{0}(x), x \in \Omega, \\
z\left(t_{k}^{+}, x\right)=z\left(t_{k}^{-}, x\right)+I_{k}\left(t, z\left(t_{k}, x\right), u\left(t_{k}, x\right)\right), x \in \Omega
\end{array}\right.
$$

where $a \geq 0$ and $b>0$ are constants, $k=1,2, \ldots, p, \Omega$ is a bounded domain in $\mathbb{R}^{N}(N \geq 1), z_{0} \in L_{2}(\Omega), \omega$ is an open nonempty subset of $\Omega, 1_{\omega}$ denotes the characteristic function of the set $\omega$, the distributed control $u$ belongs to $C\left([0, \tau] ; L_{2}(\Omega ;)\right)$ and $f, I_{k} \in C([0, \tau] \times \mathbb{R} \times \mathbb{R} ; \mathbb{R}), k=1,2,3, \ldots, p$.

Example 5.2 We believe that this technique can be applied to prove the interior controllability of the strongly damped wave equation with Dirichlet boundary conditions

$$
\begin{cases}w_{t t}+\eta(-\Delta)^{1 / 2} w_{t}+\gamma(-\Delta) w=1_{\omega} u(t, x)+f\left(t, w, w_{t}, u(t)\right), & \text { in }(0, \tau) \times \Omega, \\ w=0, & \text { in }(0, \tau) \times \partial \Omega, \\ w(0, x)=w_{0}(x), \quad w_{t}(0, x)=w_{1}(x), & \text { in } \Omega, \\ w\left(t_{k}^{+}, x\right)=w\left(t_{k}^{-}, x\right)+I_{k}^{1}\left(t, w\left(t_{k}, x\right), w_{t}\left(t_{k}, x\right), u\left(t_{k}, x\right)\right), \quad x \in \Omega, & \\ w_{t}\left(t_{k}^{+}, x\right)=w_{t}\left(t_{k}^{-}, x\right)+I_{k}^{2}\left(t, w\left(t_{k}, x\right), w_{t}\left(t_{k}, x\right), u\left(t_{k}, x\right)\right), \quad x \in \Omega, & \end{cases}
$$

in the space $Z_{1 / 2}=D\left((-\Delta)^{1 / 2}\right) \times L_{2}(\Omega), k=1,2, \ldots, p, \Omega$ is a bounded domain in $\mathbb{R}^{N}(N \geq 1)$, $\omega$ is an open nonempty subset of $\Omega, 1_{\omega}$ denotes the characteristic function of the set $\omega$, the distributed control $u \in C\left([0, \tau] ; L_{2}(\Omega)\right), \eta, \gamma$ are positive numbers and $f, I_{k}^{1}, I_{k}^{2} \in C([0, \tau] \times \mathbb{R} \times \mathbb{R} ; \mathbb{R}), k=1,2,3, \ldots, p$.

Example 5.3 Another example where this technique may be applied is a partial differential equations modeling the structural damped vibrations of a string or a beam:

$$
\left\{\begin{array}{lr}
y_{t t}-2 \beta \Delta y_{t}+\Delta^{2} y=1_{\omega} u(t, x)+f\left(t, y, y_{t}, u(t)\right), & \text { on } \quad(0, \tau) \times \Omega, \\
y=\Delta y=0, & \text { on }(0, \tau) \times \partial \Omega, \\
y(0, x)=y_{0}(x), \quad y_{t}(0, x)=y_{1}(x), & \text { in } \quad \Omega, \\
y\left(t_{k}^{+}, x\right)=y\left(t_{k}^{-}, x\right)+I_{k}^{1}\left(t, y\left(t_{k}, x\right), y_{t}\left(t_{k}, x\right), u\left(t_{k}, x\right)\right), x \in \Omega, & \\
y_{t}\left(t_{k}^{+}, x\right)=y_{t}\left(t_{k}^{-}, x\right)+I_{k}^{2}\left(t, y\left(t_{k}, x\right), y_{t}\left(t_{k}, x\right), u\left(t_{k}, x\right)\right), x \in \Omega, &
\end{array}\right.
$$

where $\Omega$ is a bounded domain in $\mathbb{R}^{n}, \omega$ is an open nonempty subset of $\Omega, 1_{\omega}$ denotes the characteristic function of the set $\omega$, the distributed control $u \in C\left([0, \tau] ; L_{2}(\Omega)\right)$ and $y_{0} \in H^{2}(\Omega) \cap H_{0}^{1}, y_{1} \in L_{2}(\Omega)$. 
Moreover, our result can be formulated in a more general setting. Indeed, we can consider the following semilinear evolution equation in a general Hilbert space $Z$

$$
\left\{\begin{array}{l}
\dot{z}=-A z+B u(t)+f^{e}(t, z, u), \quad z \in Z, \quad t \in(0, \tau], \\
z(0)=z_{0}, \\
z\left(t_{k}^{+}\right)=z\left(t_{k}^{-}\right)+I_{k}^{e}\left(t_{k}, z\left(t_{k}\right), u\left(t_{k}\right)\right), k=1,2,3, \ldots, p .
\end{array}\right.
$$

where $u \in C([0, \tau] ; U), U=Z, B_{\omega}: U \longrightarrow Z, B_{\omega} u=1_{\omega} u$ is a bounded linear operator, $I_{k}^{e}, f^{e}:[0, \tau] \times Z \times U \rightarrow Z, A: D(A) \subset Z \rightarrow Z$ is an unbounded linear operator in $Z$ with the following spectral decomposition:

$$
A z=\sum_{j=1}^{\infty} \lambda_{j} \sum_{k=1}^{\gamma_{j}}<z, \phi_{j, k}>\phi_{j, k},
$$

with the eigenvalues $0<\lambda_{1}<\lambda_{2}<\cdots<\cdots \lambda_{n} \rightarrow \infty$ of $A$ having finite multiplicity $\gamma_{j}$ equal to the dimension of the corresponding eigenspaces, and $\left\{\phi_{j, k}\right\}$ is a complete orthonormal set of eigenfunctions of $A$. The operator $-A$ generates a strongly continuous compact semigroup $\left\{T_{A}(t)\right\}_{t \geq 0}$ given by

$$
T_{A}(t) z=\sum_{j=1}^{\infty} e^{-\lambda_{j} t} \sum_{k=1}^{\gamma_{j}}<z, \phi_{j, k}>\phi_{j, k} .
$$

The control $u \in C([0, \tau] ; U)$, with $U=Z, B: Z \rightarrow Z$ is a linear and bounded operator(linear and continuous) and the functions $f^{e}, I_{k}^{e}:[0, \tau] \times Z \times U \rightarrow Z$ are smooth enough and

$$
\begin{aligned}
\left\|f^{e}(t, z, u)\right\|_{Z} & \leq \tilde{a}_{0}\|z\|_{Z}^{\alpha_{0}}+\tilde{b}_{0}\|u\|_{Z}^{\beta_{0}}+\tilde{c}_{0} \\
\left\|I_{k}^{e}(t, z, u)\right\|_{Z} & \leq \tilde{a}_{k}\|z\|_{Z}^{\alpha_{k}}+\tilde{b}_{k}\|u\|_{Z}^{\beta_{k}}+\tilde{c}_{k}, k=1,2,3, \ldots, p .
\end{aligned}
$$

In this case the characteristic function set is a particular operator $B$, and the following theorem is a generalization of Theorem 4.1.

Theorem 5.1 If vectors $B^{*} \phi_{j, k}$ are linearly independent in $Z$, then the system (5.1) is approximately controllable on $[0, \tau]$.

\section{References}

[1] J. Banas and K. Goebel, Measures of Noncompactness in Banach Spaces. Lecture Notes in Pure and Applied Mathematics, 60. Marcel Dekker, Inc., New York, 1980.

[2] D.Barcenas, H. Leiva and Z. Sivoli, A Broad Class of Evolution Equations are Approximately Controllable, but Never Exactly Controllable. IMA J. Math. Control Inform. 22, no. 3 (2005), 310-320.

[3] H. Brezis, Analisis Funcional, Teoria y Applicaciones. Alianza Universitaria Textos, Masson, Paris, 1983. Ed. cast.: Alinza Editorial, S. A., Madrid, 1984. 
[4] D. N. Chalishajar, Controllability of Impulsive Partial Neutral Funcional Differential Equation with Infinite Delay. Int. Journal of Math. Analysis, Vol. 5, 2011, Nž. 8, 369-380.

[5] Lizhen Chen and Gang Li, Approximate Controllability of Impulsive Differential Equations with Nonlocal Conditions. International Journal of Nonlinear Science, Vol.10(2010), Nž. 4, pp. 438-446.

[6] R.F. Curtain and A.J. Pritchard, Infinite Dimensional Linear Systems. Lecture Notes in Control and Information Sciences, 8. Springer Verlag, Berlin (1978).

[7] R.F. Curtain, H.J. Zwart, An Introduction to Infinite Dimensional Linear Systems Theory. Text in Applied Mathematics, 21. Springer Verlag, New York (1995).

[8] Lawrence C. Evans, Partial Differential Equations. Graduate Studies in Mathematics, Vol. 19, AMS. 1999.

[9] G. Isac, "On Rothe's Fixed Point Theorem in General Topological Vector Space", An. St. Univ. Ovidius Constanta, Vol. 12(2), 2004, 127-134.

[10] C. Kesavan, Topics in: Functional Analysis and Applications. John Wiley and Sons, 1989.

[11] H. Leiva"Controllability of a System of Parabolic equation with non-diagonal diffusion matrix". IMA Journal of Mathematical Control and Information; Vol. 32, 2005, pp. 187-199.

[12] H. Leiva and Y. Quintana, "Interior Controllability of a Broad Class of Reaction Diffusion Equations", Mathematical Problems in Engineering, Vol. 2009, Article ID 708516, 8 pages, doi:10.1155/2009/708516.

[13] H. Leiva, N. Merentes and J.L. Sanchez, "Interior Controllability of the $n D$ Semilinear Heat Equation". African Diaspora Journal of Mathematics, Special Vol. in Honor of Profs. C. Corduneanu, A. Fink, and S. Zaidman. Vol. 12, Nž. 2, pp. 1-12(2011).

[14] H. Leiva, N. Merentes and J. Sanchez "Approximate Controllability of Semilinear Reaction Diffusion" MATHEMATICAL CONTROL AND RALATED FIELDS, Vol. 2,Nž.2, June 2012.

[15] H. Leiva, N. Merentes and J. Sanchez "A Characterization of Semilinear Dense Range Operators and Applications", Abstract and Applied Analysis, Vol. 2013, Article ID 729093, 11 pages.

[16] H. Leiva, N. Merentes and J.L. Sanchez "Interior Controllability of the BenjaminBona-Mahony Equation". Journal of Mathematis and Applications, Nž 33,pp. 51-59 (2010). 
[17] A. Pazy Semigroups of Linear Operators and Applications to Partial Differential Equations., Springer-Verlag, New York, (1983).

[18] M.H. Protter, Unique continuation for elliptic equations. Transaction of the American Mathematical Society, Vol. 95, Nž 1, Apr., 1960.

[19] B. Radhakrishnan and K. Blachandran, Controllability Results for Semilinear Impulsive Integrodifferential Evolution Systems with Nonlocal Conditions., J. Control Theory Appl. 2012, 10(1), 28-34.

[20] S. Selvi and M. Mallika Arjunan, Controllability Results for Impulsive Differential Systems with Finite Delay J. Nonlinear Sci. Appl. 5 (2012), 206-219.

[21] J. D.R. Smart, Fixed Point Theorems. Cambridge University Press (1974).

[22] Xu Zhang, A Remark on Null Exact Controllability of the Heat Equation. IAM J. CONTROL OPTIM. Vol. 40, No. 1(2001), pp. 39-53.

[23] E. Zuazua, Controllability of a System of Linear Thermoelasticity,

J. Math. Pures Appl., 74, (1995), 291-315.

[24] E. Zuazua, Control of Partial Differential Equations and its Semi-Discrete Approximation. Discrete and Continuous Dynamical Systems, vol. 8, No. 2. April (2002), 469-513.

DOI: $10.7862 /$ rf.2015.8

\section{Hugo Leiva - corresponding author}

email: hleiva@ula.ve

Universidad de los Andes

Facultad de Ciencias, Departamentode Matemática

Mérida 5101-Venezuela

\section{Nelson Merentes}

email: nmerucv@gmail.com

Universidad Central de Venezuela

Facultad de Ciencias, Departamento de Matemática

Caracas -Venezuela

Received 27.03.2014 